EPJ Web of Conferences 69, 00021 (2014)

DOI: $10.1051 /$ epjconf/20146900021

(C) Owned by the authors, published by EDP Sciences, 2014

\title{
FALSTAFF: a novel apparatus for fission fragment characterization
}

\author{
Stefano Panebianco ${ }^{1}$, Diane Doré ${ }^{1}$, Fanny Farget ${ }^{2}$, François-René Lecolley ${ }^{3}$, Grégory Lehaut ${ }^{3}$, Thomas \\ Materna $^{1}$, Julien Pancin ${ }^{2}$ and Thomas Papaevangelou ${ }^{1}$ \\ ${ }^{1}$ CEA Centre de Saclay, Irfu, 91191 Gif-sur-Yvette, France \\ ${ }^{2}$ GANIL, CEA/DSM-CNRS/IN2P3, Bd H. Becquerel, 14076 Caen, France \\ ${ }^{3}$ LPC Caen, ENSICAEN, Université de Caen, CNRS-IN2P3, 14050 Caen, France
}

\begin{abstract}
The study of nuclear fission and in particular the correlation between the produced fragments and the associated neutrons is encountering renewed interest since new models are available on the market and a large set of applications show a rather stringent demand on high quality nuclear data. The future Neutrons For Science installation, being presently built at GANIL (Caen, France) in the framework of the SPIRAL2 project, will produce high intensity neutron beams from hundreds of $\mathrm{keV}$ up to $40 \mathrm{MeV}$. In view of this opportunity, the development of an experimental setup called FALSTAFF (Four Arm cLover for the Study of Actinide Fission Fragments) has been undertaken since 2011. This novel apparatus is meant to provide a full characterization of fission fragments in terms of mass, nuclear charge and kinetic energy. Moreover, it will provide a measurement of the mass before and after neutron evaporation, leading to the determination of the neutron multiplicity as a function of the fragmentation. The FALSTAFF setup is presently in its R\&D phase in order to achieve the required specifications, especially in terms of time, space and energy resolution of the different detectors.
\end{abstract}

\section{Introduction}

A regained interest in studying nuclear fission has been recently triggered by both the need to answer fundamental physics questions related to the fine structure of the process and the necessity of providing more accurate data for applications. A large number of open questions are still on the table concerning fission fragments properties. First of all, it is not fully clear how the excitation energy of the parent nucleus is transferred to the fragments and how they share this energy. In addition, the strength of the coupling between individual and collective excitations, the impact of level densities and the disappearance of shell effects with the excitation energy, the presence of even-odd effects on mass and charge yields and the spin population of the fragments are still subjects open to discussions on their interpretation and failing to be fully described by theory. In order to answer these questions, different observables are needed in a large range of fissioning systems and excitation energies. For instance, neutron multiplicity and total kinetic energy as a function of the excitation energy of the compound nucleus are needed to address the energy sharing between fragments and, more generally the properties of the scission configurations. The evolution of shell effects in the fissioning system and in the fragments could be enlighten by the study of fragment mass and isotopic yields, together with 
the correlations between the fragment energy and neutron multiplicity and the fragment mass and nuclear charge. These correlations need to be studied as a function of the excitation energy of the compound nucleus, since shell effects are supposed to disappear for high incident energy. The same can be said for the understanding of even-odd effects where the interesting observable is the evolution of the fragment yields with the compound nucleus properties (fissility, even-odd asymmetry, excitation energy). The most effective insight on the deformation energy of the fragments comes from the correlation between the total kinetic energy and the fragment mass. Finally, the study of intrinsic excitation at scission requires the measurement of scission neutrons and/or light charged particle emission. On the other hand, there is no doubt that the major application for which a precise knowledge of the fission process plays an essential role is a nuclear reactor. This is already true for the operation of the present nuclear plants, largely based on Light Water Reactors using the thermal fission of ${ }^{235} \mathrm{U}$ and ${ }^{239} \mathrm{Pu}$ isotopes. On the other hand, the large development of innovative nuclear systems, the so-called Generation IV, mostly based on fast neutrons reactors and designed to transmute nuclear wastes, requires a large effort to provide new high quality data for a large set of fissioning nuclei (from $\mathrm{Th}$ to $\mathrm{Cm})$ and in the neutron energy range going from thermal to fast $(\sim 2$ $\mathrm{MeV})$.

Based on these motivations, an exceptional opportunity is opened by the Neutrons For Science (NFS) facility being built at GANIL (Caen) in the framework of the SPIRAL 2 project [1]. Thanks to its competitive neutron flux, together with the possibility to perform experiments with radioactive targets, this neutron Time of Flight installation will allow exploration of the underexploited fast energy domain in order to provide data for fundamental physics and nuclear technology. These arguments have motivated the development of a new detection system called FALSTAFF (Four Arm cLover for the STudy of Actinide Fission Fragments) [2]. This novel apparatus is meant to provide a full characterization of fission fragments in terms of mass, nuclear charge and kinetic energy. Moreover, it will provide a measurement of the mass before and after neutron evaporation, leading to the determination of the neutron multiplicity as a function of the fragmentation. The experimental technique giving access to these observables is based on the coincidence measurement of the velocity and energy of the two fragments. The velocity is provided by a time-of-flight system while the residual energy is measured by ionization chambers. The simultaneous measurement of the energy loss within the chamber gas will also provide the nuclear charge of the light fragment. Finally, the apparatus is being designed to be eventually installed in other installations and to be possibly coupled to neutron or gamma detection systems. The FALSTAFF setup is presently in its R\&D phase in order to achieve the required specifications, especially in terms of time and space resolution of the TOF detectors. Time of flight of the fission fragments is measured in transmission mode by emissive foils coupled to secondary electron detectors (SED). A fully equipped arm, composed of two TOF detectors and an ionization chamber, is presently being tested at CEA Saclay with a spontaneous fission source. These tests are an essential step to assess and qualify our technical choices and compare the experimental results with simulations.

\section{The FALSTAFF setup}

The aim of the FALSTAFF setup is to perform a combined time of flight and residual energy measurement of complementary fission fragments in coincidence to determine their mass, nuclear charge and kinetic energy. The $2 v$ technique [3], based on the simultaneous measurement of the two fragment velocities, gives access to their masses before neutron evaporation (initial masses) under the assumption that the average fragment velocity is not modified by the evaporation process. The mass after neutron evaporation (final mass) is determined by the $E v$ technique [3] from a coincidence measurement of the fragment energy and velocity. Therefore, the mean neutron multiplicity as a function of the fragment mass may be deduced directly from the difference between initial and final mass. The velocity is provided by a time-of-flight system (TOF) while the residual energy is measured by ionization chambers (IC). The simultaneous measurement of the energy loss within the chamber gas will also provide the nuclear charge of the light fragment. 
The major challenge of the FALSTAFF setup is to provide an efficient and precise detection of low energy heavy ions in direct kinematics. These experimental conditions are clearly the most difficult since, given the low energy of ions, their energy loss through all the crossed materials is important and constitutes the main limitation to the detector resolution. These losses have to be taken into account to correctly reconstruct the velocity and energy. This implies that the thickness of material layers has to be reduced as much as possible and that the ion positions on the different layers have to be precisely measured to calculate the crossed thickness and then apply energy loss corrections. In order to achieve the desired mass resolution, the TOF and IC required performances are rather constrained. The TOF system should provide a time resolution better than $150 \mathrm{ps}$, together with a position resolution of $1.5 \mathrm{~mm}$. The energy resolution required of the IC is $1 \%$. Moreover, given the neutron flux at a $5 \mathrm{~m}$ distance from the NFS converter $\left(1.810^{6} \mathrm{n} / \mathrm{cm}^{2} / \mathrm{s}\right.$ for an incident deuteron intensity of $\left.50 \mu \mathrm{A}\right)$, an actinide target of $150 \mu \mathrm{g} / \mathrm{cm}^{2}$, a required statistics of 1000 events for the mass yields of $1 \%$ and, assuming a $1 \mathrm{MeV}$ bin size for the neutron energy, a geometrical acceptance of about $2 \%$ of $4 \pi$ is needed to allow for a beam time shorter than two weeks.

As a consequence, the use of large detectors providing low budget materials and high resolution performance is mandatory. For the TOF measurement, the necessary timing resolution will be obtained by a combination of an emissive foil and a MWPC (Multi Wire Proportional Counter) as secondary electrons detector (SED) [4]. A large area can be covered with this type of detector without degrading the time and spatial resolution. The most recent results on the R\&D on two prototype SEDs are presented in the next section. The fragment energy will be measured with ionization chambers, this type of detector being able to reach the desired 1\% energy resolution [5]. The fragment nuclear charge identification will be provided by $\Delta \mathrm{E}-\mathrm{E}$ correlation up to $\mathrm{Z}=40$. Among the different types of existing ionization chamber setups, segmented or axial chambers are foreseen. In addition, a more advanced detector, providing the collection of the scintillation photons generated by ions crossing the gas, is under study [6]. This latter solution could provide a fast time signal in addition to the energy measurement. This could allow elimination of the time-stop detector. Since this innovative R\&D is still in progress, an axial chamber, not presented here, will be constructed for the first version of the setup.

Figure 1. Schematic view of the two-arm FALSTAFF setup.

The first version of the setup will be composed of one arm consisting of two SED detectors together with an existing ionization chamber (called MiniHarpee). This setup will be tested with a ${ }^{252} \mathrm{Cf}$



spontaneous fission source in order to measure the final mass of light fragments (the heavy ones cannot be reconstructed with this setup because of their large nuclear charge). In a second step, a second arm will be added in order to realize the coincidence 2v and Ev techniques (Figure 1). For this setup, two axial ionization chambers will replace the MiniHarpee. A spectroscopic ${ }^{252} \mathrm{Cf}$ source, providing the two fission fragments, is presently under development specifically for this setup. It will first allow verification of the resolutions obtained for the determination of the initial and final mass of light and heavy fragments. Then the setup will be moved to NFS for a day-one experiment using a ${ }^{238} \mathrm{U}$ target. In a last step, the solid angle of the FALSTAFF setup will be increased by adding two other arms thus allowing the collection of better statistics or the use of thinner targets. 


\subsection{The R\&D on SED}

The TOF measurement is provided by a system of two SEDs, one to measure the time of start and the other for the stop. A SED system is made of an emissive foil and a MWPC detector (Figure 2) [4]. A aluminized Mylar ( $0.9 \mu \mathrm{m}$ thickness), inclined at $45^{\circ}$ with respect to the horizontal direction and polarized at $-10 \mathrm{kV}$, is placed along the path of the fission fragments. A grounded grid placed at $1 \mathrm{~cm}$ from this emissive foil provides a high electric field close to the foil. The secondary electrons extracted by the crossing ion from the Mylar foil are then accelerated to $10 \mathrm{keV}$ and fly towards the MWPC detector. The accelerated electrons pass through the MWPC entrance foil $(0.9 \mu \mathrm{m}$ aluminized Mylar) with $70 \%$ efficiency. They produce ionization electrons inside the gas which are then amplified while drifting towards the cathode. The amplification takes place over the whole drift path thanks to the low pressure of the gas (4-6 torr). The time signal is then read from the wire plane by a fast ampli fier. The spatial information is extracted from the charge induced on a 2D pixelized cathode read by a high frequency sampling Front End Electronics card based on the AFTER chip [7].

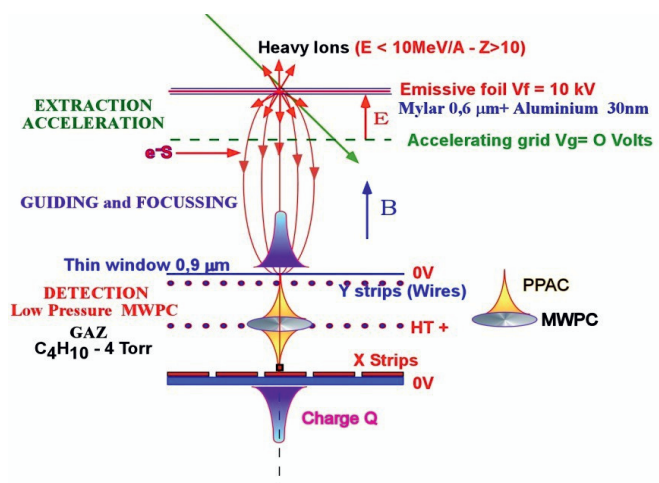

Figure 2. Working principle of a SED detector.

A MWPC-SED prototype of $15 \mathrm{~cm}$ x $20 \mathrm{~cm}$ active area with a pixelized cathode (68x48 pads) and a gap of $1.6 \mathrm{~mm}$ between the entrance window and the anode has been built and tested in laboratory with fission fragments from a $252 \mathrm{Cf}$ source. Both time and position resolutions have been measured [2]. The time resolution as a function of the voltage applied between the entrance window and the anode is shown for different pressures in the left panel of Figure 3. The best obtained resolution is 120 ps but further improvements are expected from noise reduction, possibly improving the resolution down to $100 \mathrm{ps}$. The position resolution of the SED depends on the focalization of the secondary electrons reaching the MWPC entrance window. Therefore, the SED has been placed inside a dipole providing a magnetic field up to 150 gauss. The right panel of Figure 3 shows the position resolution in the $\mathrm{X}$ and $\mathrm{Y}$ directions as a function of the magnetic field. A resolution of $1.5 \mathrm{~mm}$ is reached for a field of 90 gauss. The achieved time and position resolutions are already satisfactory since they correspond rather well to the needed specifications. Moreover, the position resolution requirements on the start SED being less demanding, this detector does not need to be placed inside a magnetic field (Figure 1). The actual experimental values of time and position resolutions are used in the simulation of the full setup. 

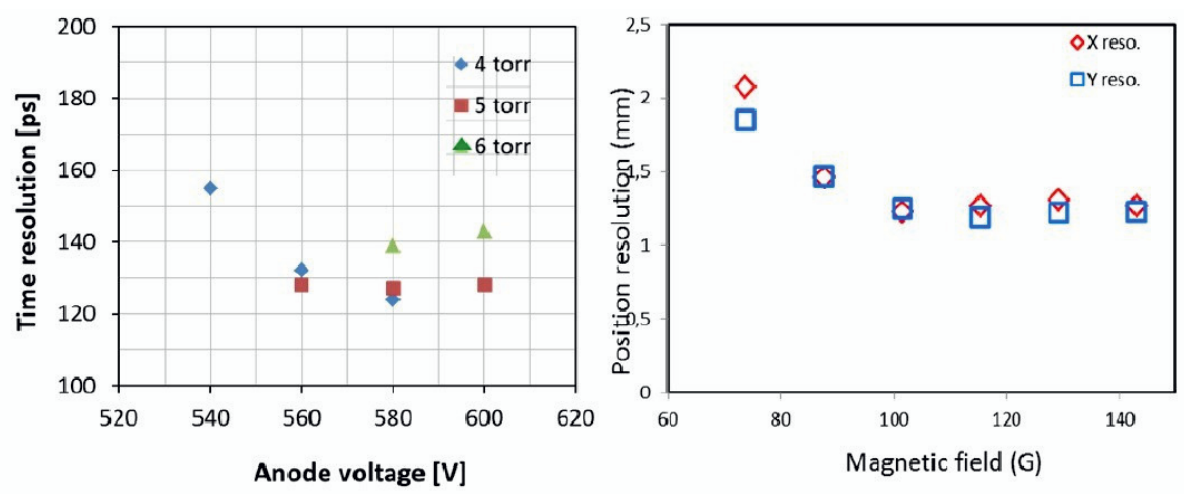

Figure 3. Time resolution as a function of the anode voltage for different gas pressures (left). Position resolution in the $\mathrm{X}$ and $\mathrm{Y}$ directions as a function of the magnetic field (right).

\subsection{The R\&D on IC}

The design of an axial ionization chamber, providing a high $\Delta \mathrm{E}$ and $\mathrm{E}$ resolution measurement and covering a large solid angle, is presently ongoing. Two major constraints limit the needed energy resolution of $1 \%$. First of all, the entrance window of the IC should be large enough to cover the needed acceptance, keeping a thin and uniform thickness. Moreover, given the differential pressure the window has to stand (around 20 mbar inside the IC and secondary vacuum outside), its bending has to be reduced to avoid a ballistic deficit. For the moment, the foreseen solution is a polypropylene window of $0.9 \mu \mathrm{m}$ thickness. For the FALSTAFF preliminary test, the existing MiniHarpee IC from GANIL is being used.

A more fancy possibility is under R\&D with the aim of providing an IC capable of performing a combined measurement of energy and time. This IC is based on the scintillation properties of the IC gas and is meant to collect by a PMT the photons generated by ions crossing the gas [6]. This solution would provide a fast time signal in addition to the energy measurement, which will allow elimination of the time-stop detector. Preliminary tests have been performed on an IC prototype coupled to 2 PMT and using $\mathrm{CF}_{4}$ and $\mathrm{N}_{2}$ gas at different pressures. An energy resolution of $1.2 \%$ has been obtained with $\mathrm{CF}_{4}$ at 250 mbar, together with a time resolution of $200 \mathrm{ps}$. Although these values do not fulfill FALSTAFF specifications, they are very encouraging since the identified margin of improvement is rather high.

\section{Simulation of the setup}

In order to assess precisely the feasibility of the measurement a full and detailed simulation of the FALSTAFF two-arm setup (Figure 1) has been performed using the GEANT4 Monte-Carlo code [8]. The simulation activity also allows developing the analysis method and optimizing the achievable mass and charge resolutions taking into account the different layers of material and the position, time and energy resolutions of the different detectors. To reconstruct initial and final mass of both fission fragments from their velocity and energy, the analysis is based on an event-by-event iteration procedure. This procedure was first developed for events simulated without any energy straggling or experimental resolution. Indeed, assuming that the nuclear charge of the light ion is known (which implies the knowledge of the heavy one from charge conservation), the energy loss corrections are applied to each ion from the crossed material thickness. Then, the initial and final masses of the two fragments are reconstructed from the simulated velocities and energies. As an example, the initial and final masses of two typical fission fragments in coincidence $\left({ }^{140} \mathrm{Cs}\right.$ and $\left.{ }^{98} \mathrm{Rb}\right)$ are correctly reconstructed with a mass resolution better than $0.01 \mathrm{amu}$, coming only from the energy loss correction uncertainty in the simulation. With the addition of the energy straggling and the experimental resolutions, we ob- 
tain a resolution better than $1 \mathrm{amu}$ for the initial mass and $1.9 \mathrm{amu}$ (1.6 amu) for the heavy (light) fragment final mass.

For the simulation of the whole fission fragments mass distribution, the ${ }^{252} \mathrm{Cf}(\mathrm{sf})$ initial mass yields, together with the mean neutron multiplicities, have been generated using the GEF code [9]. Then, the reconstructed initial and final mass distributions are obtained with and without including in the simulation the energy straggling and the experimental resolutions. The two results are shown in Figure 4 (without and with energy straggling and experimental resolutions in the left and right panels respectively). As expected, the energy straggling and the experimental resolutions have the effect of smearing out the reconstructed mass distributions. Nevertheless, when the evaporation process is included, the feasibility of extracting the neutron mean multiplicity as a function of the initial mass could be demonstrated. In the analysis of the simulated data, the neutron multiplicity is determined on an event-by-event basis. Then, for a given initial mass, the average neutron multiplicity $\left.\left(<A_{i}-A_{f}\right\rangle\right)$ and its uncertainty $\left(\left\langle A_{i}-A_{f}\right\rangle N_{N}\right)$ are calculated over $N$ events. The reconstructed neutron multiplicity, together with the simulated neutron multiplicity, is shown in Figure 5. One can clearly see that the extracted correlation is very satisfactory. The lack of statistics in the wings and the symmetric valley of the mass distribution lead to larger uncertainties and some discrepancies between simulated and reconstructed values. We observe also a steeper slope for reconstructed light fragments that has to be investigated further.
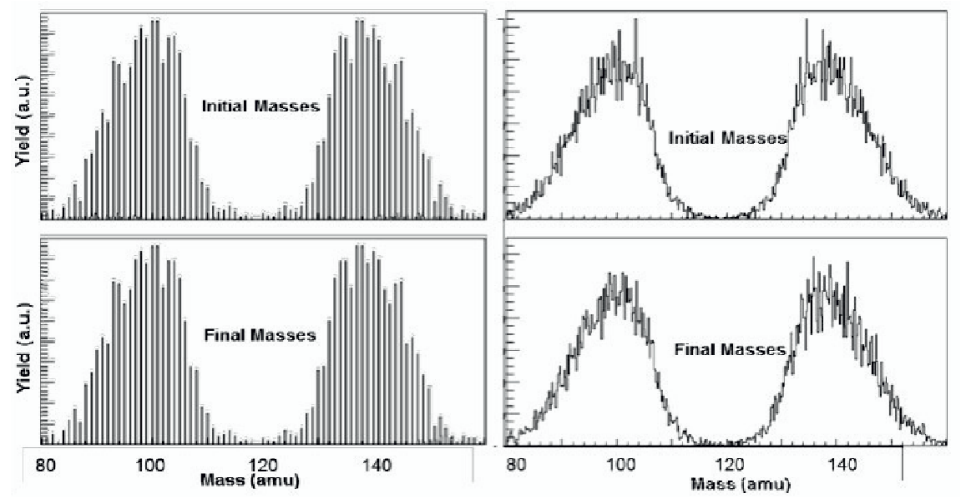

Figure 4. Initial (top range) and final (bottom range) fragment mass distributions from ${ }^{252} \mathrm{Cf}(\mathrm{sf})$ without (left column) and with (right column) energy straggling and detection resolutions.

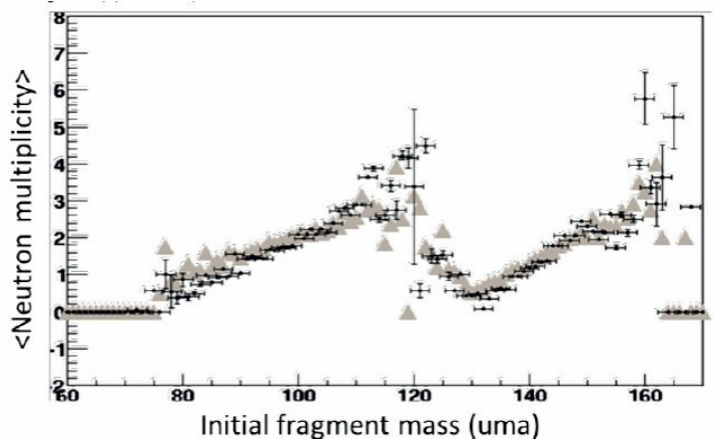

Figure 5. Mean neutron multiplicity as a function of the initial fragment mass. The grey triangles represent the simulated multiplicity while the cross represents the reconstructed one. 


\section{Conclusion and perspectives}

A new experimental apparatus, called FALSTAFF and devoted to the full characterization of fission fragments is being developed. In addition to measuring the masses before and after neutron evaporation, the nuclear charge and the kinetic energy of both fragments in coincidence, it will give access to the mean neutron multiplicity associated with each fragment. The detailed simulation of the setup has proven the feasibility of the measurement with the already achieved detector resolution. The R\&D activity on both the SED detectors and IC shows that the time, position and energy resolution requirements are fulfilled. The first tests on a one-arm setup (Figure 6) are in progress with a ${ }^{252} \mathrm{Cf}(\mathrm{sf})$ source. The mid-term plans foresee the installation of a two-arm setup, eventually equipped with scintillation-based IC, to be fully tested by 2015 with a spectroscopic $\mathrm{Cf}$ source. Then, once the commissioning of the NFS installation is completed, the FALSTAFF setup will be moved to GANIL for a Day-One experiment with a ${ }^{238} \mathrm{U}$ target. Finally, a full experimental program at NFS is foreseen, eventually with a $2 \mathrm{x}$ two-arm setup, to explore fission fragment characteristics of major and minor actinides as a function of the neutron energy in the range $0.5-5 \mathrm{MeV}$.
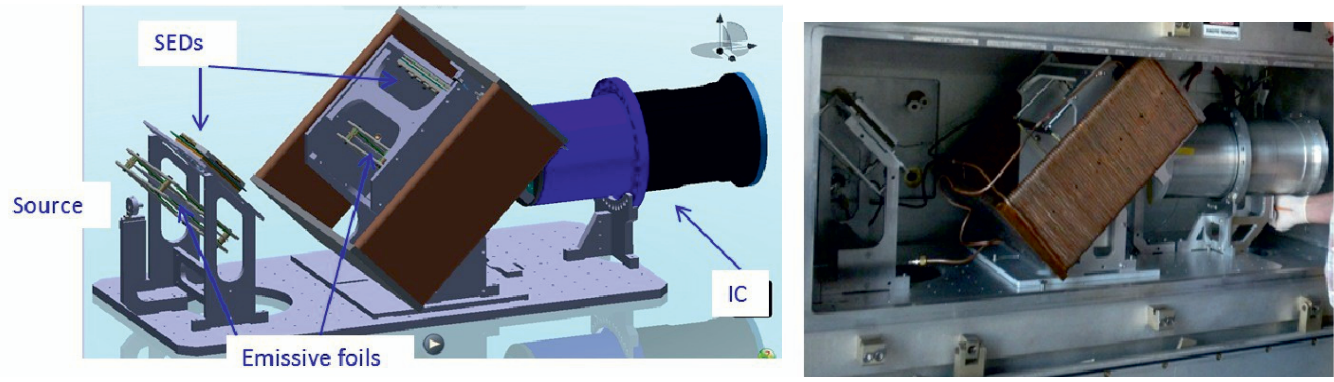

Figure 6. Design of the FALSTAFF one-arm setup (left) and picture of the present installation (right).

\section{References}

1. X. Ledoux et al., Proc. « Nuclear Data for Science and Technology 2013», New York (USA), 4$8 / 03 / 2013$

2. D. Doré et al., Proc. «Fission 2013», Caen (France), 28-31/05/2013

3. H. C. Britt et al., Instr. Meth. 24, 13 (1963)

3. J. Pancin et al., Journ. Instr. Sc. Tech. 4, 120012 (2009)

4. J.P. Bocquet et al., Nucl. Instr. Methods A 267, 466 (1988)

5. F. R. Lecolley et al., Proc. « XVIII ${ }^{\text {th }}$ Colloque Ganil », Port-en-Bessin (France), 23-27/09/2013

6. P. Baron et al., IEEE TNS 55-3, 1744 (2008)

7. R. Brun et al., Computer Physics Communications, 180-12, 2499 (2009)

9. K.-H. Schmidt, B. Jurado, JEF/DOC 1423, OECD Nuclear-Energy Agency, Paris (2012) 